Nicolas Schmuziger • John Allum

Carlos Buitrago-Téllez $\cdot$ Rudolf Probst

\title{
Incapacitating hypersensitivity to one's own body sounds due to a dehiscence of bone overlying the superior semicircular canal. A case report
}

Received: 9 December 2004 / Accepted: 15 December 2004 / Published online: 21 June 2005

(C) Springer-Verlag 2005

\begin{abstract}
We present a case study of a 49-year-old patient with an 8-year history of hypersensitivity to sound produced by intrinsic but not extrinsic sources. Findings that indicated an organic problem were: a supranormal bone conduction threshold of -25 to $-15 \mathrm{~dB}$ HL from 0.25 to $1 \mathrm{kHz}$ with an air-bone gap of 15 to $45 \mathrm{~dB} \mathrm{HL}$, a lower threshold and larger amplitude for vestibularevoked myogenic potentials, eye movement reactions to sound and trunk pitch sway in response to sound. Results of immitance audiometry and otoacoustic emission testing were within normal limits and indicative of intact middle ear conductance. A high-resolution CT scan of the temporal bone demonstrated a dehiscence of bone overlying the superior semicircular canal. These findings support previous research indicating that auditory energy reaches the cochleo-vestibular receptor systems more easily via transmission through cerebrospinal fluid than through bone. Therefore, a dehiscence of the bone overlying the superior semicircular canal may lead to hypersensitivity to intrinsic sound. We recommend that similar findings in other patients be followed up with an evaluation of middle ear function and the temporal bone with high-resolution CT scan.
\end{abstract}

Keywords Dehiscence - Superior semicircular canal dehiscence - Bone conduction thresholds - VEMP . Balance control

\footnotetext{
N. Schmuziger $\cdot$ J. Allum $\cdot$ R. Probst

Department of Otorhinolaryngology, University Hospital, Basel, Switzerland

C. Buitrago-Téllez

Department of Radiology, University Hospital,

Basel, Switzerland

N. Schmuziger $(\bowtie)$

Department of Otorhinolaryngology, Cantonal Hospital,

5001 Aarau, Switzerland

E-mail: nicolas.schmuziger@ksa.ch

Tel.: + 41-62-8384760

Fax: + 41-62-8385109
}

\section{Introduction}

Dehiscence of bone overlying the superior semicircular canal or superior semicircular canal dehiscence (SSCD) is a recently recognized condition with vestibular symptoms and signs typically provoked by sound and/or pressure stimuli $[3,4,6,12,13]$. However, it has become apparent that some patients with SSCD have auditory manifestations with or without vestibular signs or symptoms [9, 11, 14]. These patients may have a hypersensitivity to their own body sounds such as hearing their eye movements or blood pulse $[13,20]$. This hypersensitivity may be assessed by bone vibrator threshold measurements. However, use of standard clinical audiometers may not be appropriate because minimal test tone levels are usually restricted to $-10 \mathrm{~dB}$ HL.

We present the case of a patient with incapacitating hypersensitivity to his own body sounds, which was assessed by bone vibrator thresholds at test tone levels of -15 to $-25 \mathrm{~dB} \mathrm{HL}$ in the lower frequency range as well as vestibular tests. These findings underscore the importance of accurate assessment of audiometric bone vibrator thresholds to levels well below $-10 \mathrm{~dB} \mathrm{HL}$ in patients with complaints of hypersensitivity to their own body sounds.

\section{Case report}

A 49-year-old male patient reported experiencing severe hypersensitivity to his own body sounds for 8 years. Examples were sounds produced by his own voice, digestion, walking and running, and even eye movements. During meals, he heard his mastication sounds so loudly that he did not understand the conversation of the persons sitting opposite him. The same was true when he spoke. This hypersensitivity was very disturbing, and more and more he avoided social activities that required the use of his own voice. Furthermore, he suffered from short duration vertigo (ca. $1 \mathrm{~s}$ ) induced by 
humming (Tullio's phenomenon). In these moments, his surroundings tilted upwards and, occasionally, he felt he was moving backwards.

The otoscopy was normal, and tuning fork tests showed a normal Weber, but a negative Rinne on both ears. The pure-tone air conduction thresholds at all standard frequencies from 0.25 to $8 \mathrm{kHz}$, including inter-octave frequencies of 1.5 and $3 \mathrm{kHz}$, were measured with reference to ISO 389 using a digital, PC-controlled audiometer (Insider of Audiocare, Switzerland) equipped with circumaural Sennheiser HDA 200 earphones. The bone vibrator was calibrated in such a way that measurements could be performed down to $-40 \mathrm{~dB}$ HL. The audiometer was calibrated immediately before audiological testing. Audiometric results are shown in Fig. 1. Air conduction thresholds in both ears were normal with 0 to $20 \mathrm{~dB}$ HL between 0.25 to $4 \mathrm{kHz}$. In contrast, bone vibrator thresholds were "supranormal" with -15 to $-25 \mathrm{~dB} H \mathrm{HL}$ in the frequency range 0.25 to $1 \mathrm{kHz}$, where an "air-bone" gap of 15 to $30 \mathrm{~dB}$ was found. Results of immitance audiometry and clickevoked otoacoustic emission tests were within normal limits. Routine clinical neurootological and electronystagmography examinations yielded normal results. The examination under Frenzel glasses showed no nystagmus after Valsalva maneuvers.

Vestibular-evoked myogenic potentials (VEMP) were recorded following the recommendations of Akin et al. and Cheng et al. [1, 5]. The intensities of the $500-\mathrm{Hz}$ tone bursts ranged 60-100 dB nHL. EMG responses from the sterno-cleidomastoid (SCM) neck muscles were averaged over a series of 500 stimuli, and each series was repeated twice. EMG responses from each side were amplified, bandpass-filtered from 20 to $3,000 \mathrm{~Hz}$ and averaged by a Nicolet Spirit Lite (Nicolet Biomedical Inc, Madison, Wis.). Analysis time was 100 ms. Off-line, the mean peak latency and the peak-to-peak amplitude between the peaks p13 and n 23 were measured.

VEMPs were present bilaterally in SCM muscles in response to the standard $100-\mathrm{dB}$ nHL tone burst stimulus. The ipsilaterally elicited VEMP amplitude was $150 \mu \mathrm{V}$ on the right and $188 \mu \mathrm{V}$ on the left ear. The VEMP threshold was $65 \mathrm{~dB}$ nHL on both sides (Fig. 2).

Pulsatile test tones of the audiometer at high intensities presented to the ear by earphone induced visible head movements directed forward and to the stimulated ear. These involuntary head movements were clearly induced by contractions of the ipsilateral sternocleidomastoid muscle synchronous to the stimulus. The corresponding test-tone thresholds with barely visible head movements at $0.25,0.5$ and $1 \mathrm{kHz}$ are shown in Fig. 1.

Results of video-oculography (VOG) are shown in Fig. 3. With visual fixation, eye movements were not detected either in the vertical or horizontal plane. In darkness, without any fixation point, a left-beating horizontal spontaneous nystagmus was observed. Humming a low-pitched tone with his lips closed, the patient developed slow $(1 / 4 \mathrm{~Hz})$ undulating eye movements in the vertical plane and irregular eye movements in the horizontal plane.

Balance control during two-legged stance on foam was quantified using simple measurements of trunk sway with body-worn angular velocity transducers [2, 8]. Amplitudes of trunk sway angle and angular velocity in the roll and pitch directions were recorded. Tasks were performed with and without presentations of pure tones of $100 \mathrm{~dB} \mathrm{HL}$ at $500 \mathrm{~Hz}$ by earphones to the subjectively more affected right ear. With no acoustic stimulation, balance control was within normal limits. However, reproducible sound-induced forward pitching trunk movements were recorded some 1-2 s from the onset of the pure tone presentation (see Fig. 4).
Fig. 1 Air-conduction and bone vibrator thresholds for the right and left ear on separate panels. Additionally, pulsatile test tone thresholds inducing barely visible head movements are shown in the lower part of the audiograms

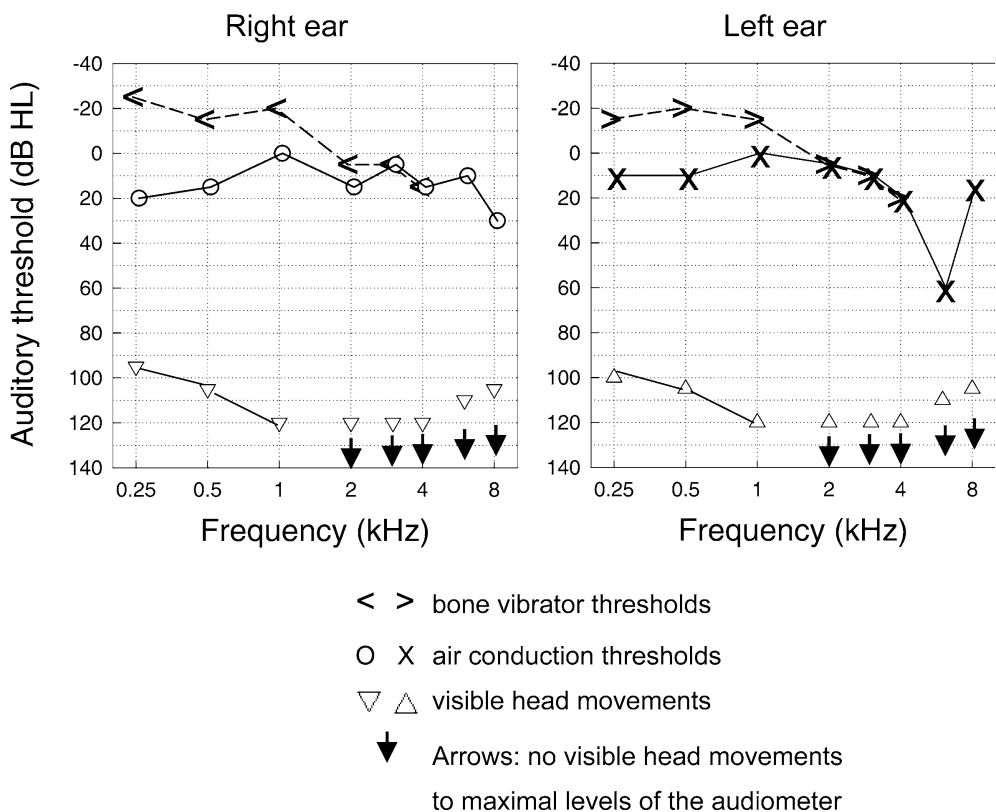


Fig. 2 Air-conducted vestibular-evoked myogenic potentials. Two similar responses have been averaged. $R t$ indicates electromyographic responses for the right sternocleidomastoid (SCM) muscle to right ear stimulation. $L t$ indicates responses for the left sternocleidomastoid muscle to left ear stimulation. Positive ( $p 13)$ and negative (n23) peak VEMP were recorded in each ipsilateral SCM to short tone bursts. $Y$-axis: 1 unit represents $24.8 \mu \mathrm{V}$. X-axis: 1 unit represents $10 \mathrm{~ms}$. VEMP are depicted for stimuli of $100 \mathrm{~dB}$ HL and $70 \mathrm{~dB}$ HL. VEMP were not present at $60 \mathrm{~dB} H \mathrm{HL}$
Rt.

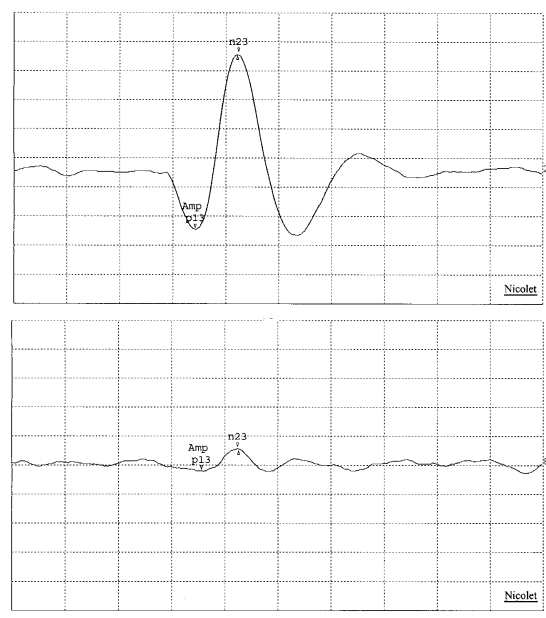

Lt.

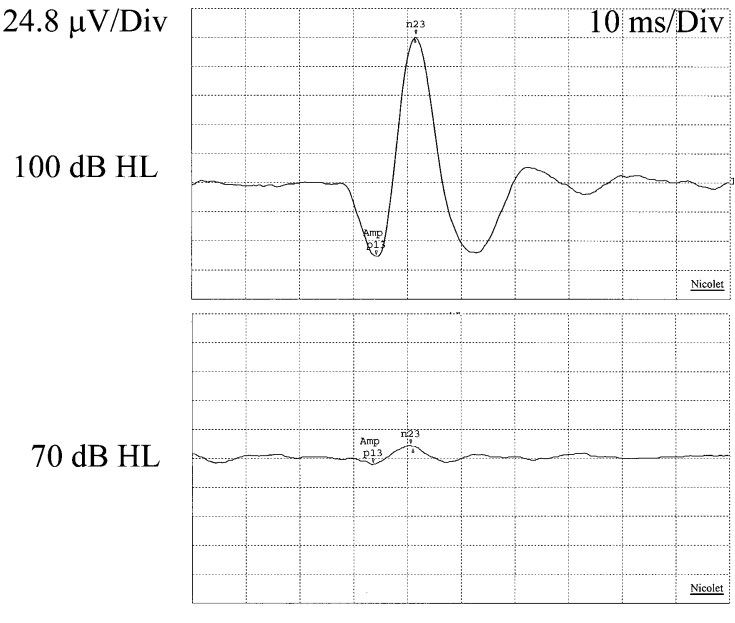

$10 \mathrm{~ms} /$ Div

High resolution computed tomography of the temporal bone demonstrated clearly a dehiscence of bone overlying the superior semicircular canal on both sides, as illustrated in Fig. 5 using a 3-D reconstruction of the skull base. The 2D coronal reconstructions parallel to the superior semicircular canal confirmed the findings of the $3 \mathrm{D}-\mathrm{CT}$ reconstructions.

\section{Discussion}

The main symptom in this case with SSCD was an incapacitating hypersensitivity to his own body sounds, which was verified with pure tone audiometry. A "hypersensitivity" of bone vibrator thresholds was revealed despite the normal or nearly normal air conduction thresholds. Two mechanisms have been proposed by Mikulec et al. [11] for an "air-bone gap": (1) shunting air-conducted sound away from the cochlea, thus elevating air-conduction thresholds and (2) increasing the difference in impedance between the oval and round windows, thus improving thresholds for "bone-conducted" sound. However, a third mechanism based on a fluid model of "bone" conduction must be considered. Sohmer and colleagues [7, 17] have presented evidence that acoustic energy reaches the cochlea probably more by cerebrospinal fluid (CFS) transmission than by bone actually vibrating. Therefore, the term "bone vibrator thresholds" is probably more appropriate than "bone conduction thresholds." This fluid pathway could easily explain the hypersensitivity of bone vibrator thresholds in the presence of a dehiscence of the bone overlying the superior semicircular canal. Such a pathway also may explain some findings in other pathologies such as enlarged vestibular aqueduct or cochlear dyplasias with an abnormal communication between the inner ear and the
Fig. $3 V O G$ Vertical and horizontal positions of the right eye are plotted against time. The top traces were recorded with visual suppression of nystagmus and the middle traces without visual suppression, both without sound or pressure stimulation. The bottom traces were recorded without visual suppression of nystagmus but with sound stimulation, produced by humming of the patient

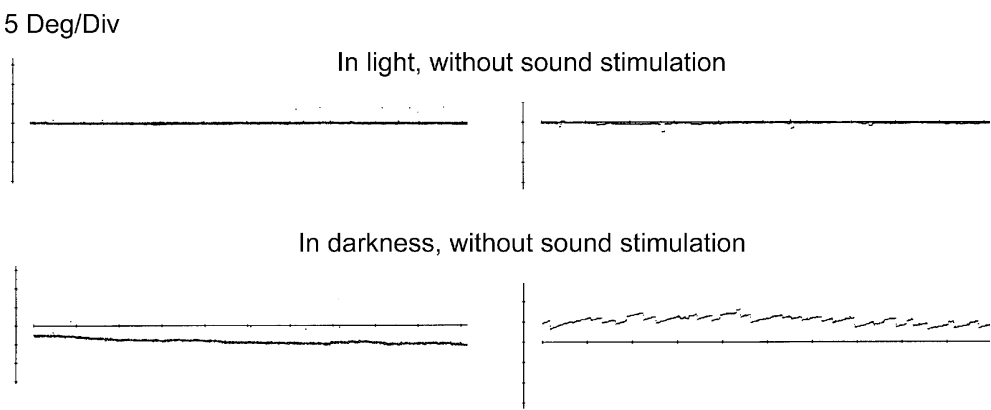

In darkness, with sound stimulation
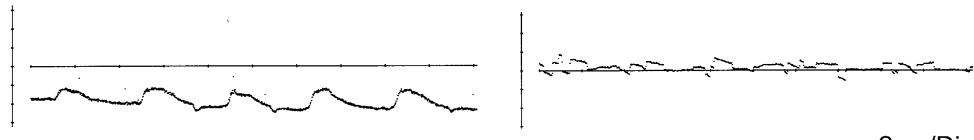
Fig. 4 Trunk sway deviations, patient standing on both legs on a foam support surface. The pitch and roll deviations are shown during presentation of a pulsatile pure-tone of $100 \mathrm{~dB}$ HL at $0.5 \mathrm{kHz}$ through earphones to the right ear over a time of 20 s. Top panel: testing with eyes closed. Bottom panel: testing with eyes open
Stimuli with $100 \mathrm{~dB} \mathrm{HL}$ tone at $500 \mathrm{~Hz}$

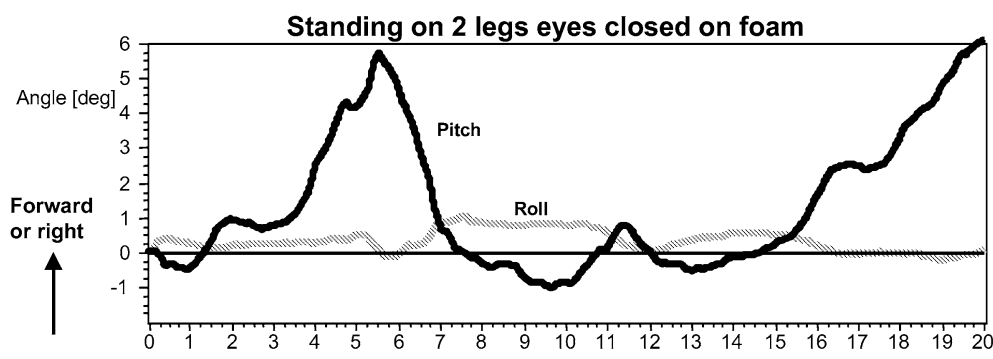

Standing on 2 legs eyes open on foam

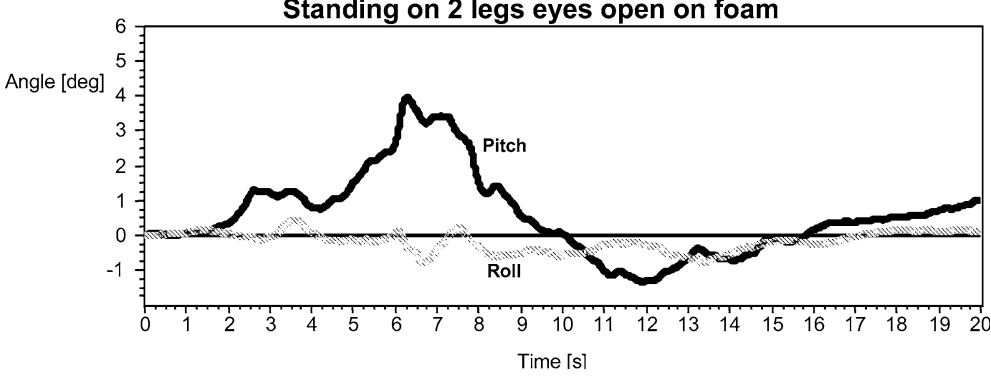

cerebrospinal fluid. Furthermore, Sohmer et al. demonstrated recently in the rat that semicircular canal fenestration improved bone- but not air-conducted auditory thresholds [18].

Mikulec et al. have stated that many patients with SSCD had undergone middle ear surgery without experiencing a benefit [11]. Therefore, the recognition of this pathology is important to avoid unnecessary surgery. "Hypersensitivity" of bone vibrator thresholds together with normal or elevated low-frequency thresholds obtained through earphones should lead to careful audiological evaluation of middle ear function and to an evaluation with high resolution computed tomography of the temporal bone. "Conductive losses" should be confirmed by objective measures such as absence of acoustic stapedius reflexes and/or otoacoustic emissions. The radiological evaluation should routinely include the specific question of a dehiscence of a semicircular canal. For this purpose, high resolution CT of the temporal bone using the multidetector technique allows high quality $2 \mathrm{D}$ and $3 \mathrm{D}$ reconstructions in a dedicated projection [10]. In this context, 2D reconstructions along the semicircular canals are mandatory. 3D reconstructions are useful for demonstrating the localization of the dehiscense, but the careful use of an adequate threshold value (150-200 Hounsfield units) to avoid pseudoforamina or bone loss should be taken into account [15]. In any case, a direct correlation with the $2 \mathrm{D}$ reconstructions is necessary for diagnosis.

This case with SSCD was marked by the patient's complaints of incapacitating hypersensitivity to his own body sounds, which was confirmed by adequate assessment of audiometric bone vibrator thresholds to levels well below $-10 \mathrm{~dB}$ HL. Treatment may include a middle fossa approach with craniotomy for resurfacing of the canal dehiscence [13]. Eight cases have been reported with SSCD, all of whom underwent surgical treatment $[3,13]$. Two of them had some sensorineural hearing loss after this treatment. However, both patients were treated by plugging the superior semicircular canal. Resurfacing may be the preferred technique to avoid hearing loss. Neurosurgical complications were not reported.

Our patient had lower thresholds and higher amplitudes for VEMP recorded from both ears in comparison to findings in normal ears. These findings are in agreement with the literature $[3,19]$ and support the theory of a "third window" that allows volume and pressure displacements, and thus larger deflections of the vestibular sensors, thereby causing the vestibular organ to be more responsive to sound and pressure changes [16].

In our patient, intense low-pitched humming produced illusions of head movements in the vertical upward direction. During such a stimulus, eye movements undulated, but no nystagmus was present in the vertical plane. According to Cremer et al., superior canal activation is characterized by nystagmus with upward torsional slow phases [6]. They investigated 11 patients with SSCD using three-dimensional scleral search coils, and 10 out of these 11 patients developed such nystagmus when loud tones were presented to the affected ear or when the patient performed a Valsalva maneuver. We did not measure torsional eye movement in our patient and cannot confirm if his eye movements were the product of superior canal activation or not.

Chronic dysequilibrium is a common complaint of patients with SSCD [11, 13, 14]. However, balance control was not quantified in these patients. Our measurements of trunk sway demonstrated reproducible forward directed trunk movements in the pitch plane with a latency of a few seconds after the onset of a low frequency pure tone stimulus at high levels. The direction of these movements is consistent with the subjective 
Fig. 5 High resolution computed tomography of the temporal bone in $0.75-\mathrm{mm}$ slides and reconstruction with respect to the anatomy of the superior semicircular canal. a 3D-CT reconstruction with the surface rendering technique showing the dehiscence on both superior semicircular canals, b $2 \mathrm{D}$ reconstruction parallel to the superior semicircular canal confirms the missing bone in the superior aspect of the canal on the left and $\mathbf{c}$ on the right petrous bone

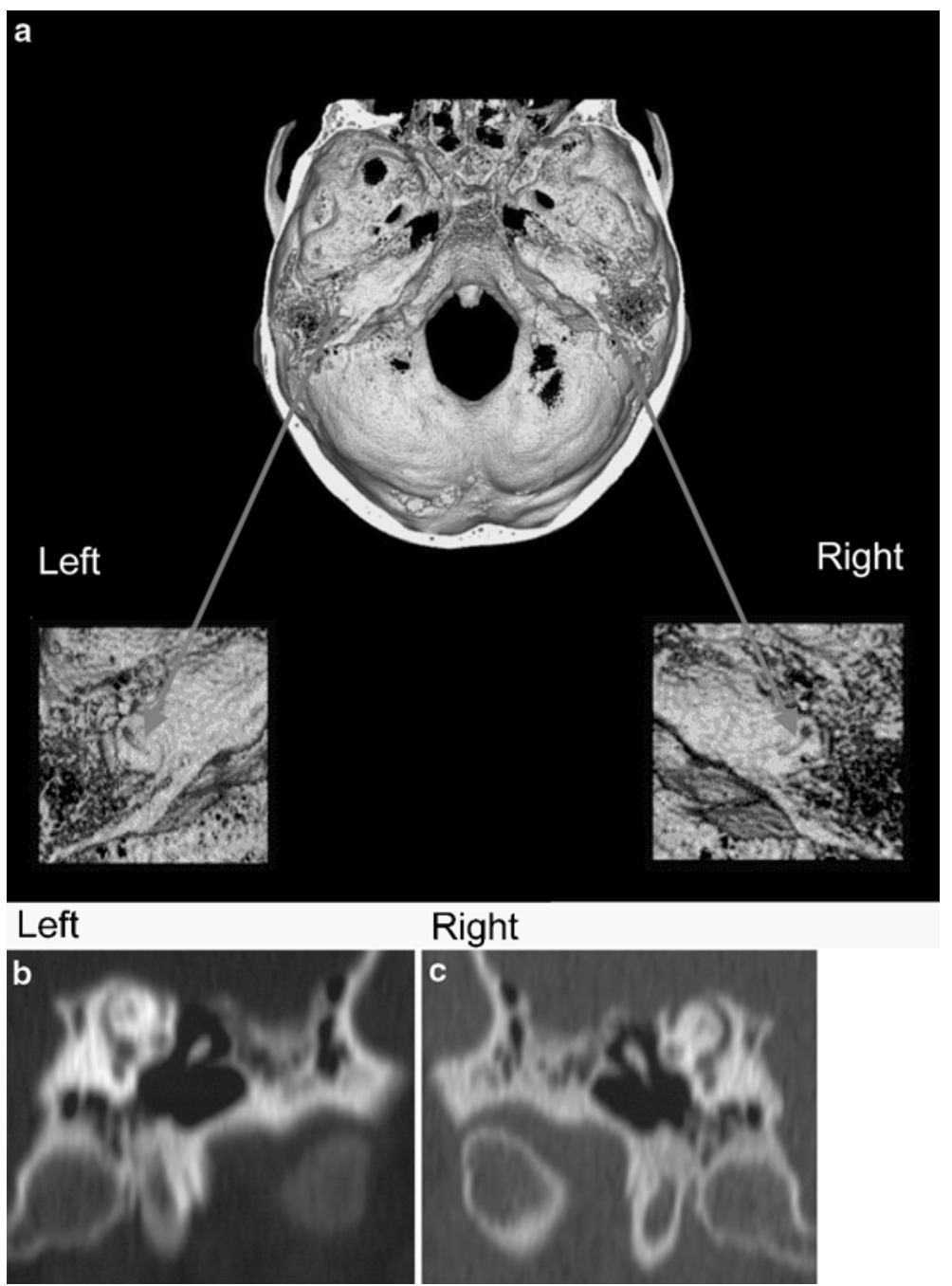

motion elicited when he hummed and with stimulation of the vertical canal system.

In conclusion, hypersensitivity to one's own body sounds may be a predominant and incapacitating symptom in patients with SSCD, and accurate assessment is crucial.

Acknowledgements Dr. F. P. Harris provided very helpful comments on an earlier version of this manuscript.

\section{References}

1. Akin FW, Murnane OD, Proffitt TM (2003) The effects of click and tone-burst stimulus parameters on the vestibular evoked myogenic potential (VEMP). J Am Acad Audiol 14:500-509

2. Allum JH, Adkin AL (2003) Improvements in trunk sway observed for stance and gait tasks during recovery from an acute unilateral peripheral vestibular deficit. Audiol Neurootol $8: 286-302$

3. Brantberg K, Bergenius J, Mendel L, Witt H, Tribukait A, Ygge J (2001) Symptoms, findings and treatment in patients with dehiscence of the superior semicircular canal. Acta Otolaryngol 121:68-75

4. Carey JP, Minor LB, Nager GT (2000) Dehiscence or thinning of bone overlying the superior semicircular canal in a temporal bone survey. Arch Otolaryngol Head Neck Surg 126:137-147
5. Cheng PW, Murofushi T (2001) The effects of plateau time on vestibular-evoked myogenic potentials triggered by tone bursts. Acta Otolaryngol 121:935-938

6. Cremer PD, Minor LB, Carey JP, Della Santina CC (2000) Eye movements in patients with superior canal dehiscence syndrome align with the abnormal canal. Neurology 55:1833-1841

7. Freeman S, Sichel JY, Sohmer H (2000) Bone conduction experiments in animals - evidence for a non-osseous mecha-

8. Gill J, Allum JH, Carpenter MG, Held-Ziolkowska M, Honegger F, Pierchala K (2001) Trunk sway measures of postural stability during clinical balance tests: Effects of age. J Gerontol $56: 438-447$

9. Halmagyi GM, Aw ST, McGarvie LA, Todd MJ, Bradshaw A, Yavor RA, Fagan PA (2003) Superior semicircular canal dehiscence simulating otosclerosis. J Laryngol Otol 117:553-557

10. Howard JD, Elster AD, May JS (1990) Temporal bone: threedimensional CT. Part II. Pathologic alterations. Radiology 177:427-430

11. Mikulec AA, McKenna MJ, Ramsey MJ, Rosowski JJ, Herrmann BS, Rauch SD, Curtin HD, Merchant SN (2004) Superior semicircular canal dehiscence presenting as conductive hearing loss without vertigo. Otol Neurotol 25:121-129

12. Minor LB, Solomon D, Zinreich JS, Zee DS (1998) Soundand/or pressure-induced vertigo due to bone dehiscence of the superior semicircular canal. Arch Otolaryngol Head Neck Surg $124: 249-258$

13. Minor LB (2000) Superior canal dehiscence syndrome. Am J Otol 21:9-19 nism. Hear Res 146:72-80 
14. Minor LB, Carey JP, Cremer PD, Lustig LR, Streubel SO, Ruckenstein MJ (2003) Dehiscence of bone overlying the superior canal as a cause of apparent conductive hearing loss. Otol Neurotol 24:270-278

15. Reisser C, Schubert O, Forsting M, Sartor K (1996) Anatomy of the temporal bone: detailed three-dimensional display based on image data from high-resolution helical CT: a preliminary report. Am J Otol 17:473-479

16. Sheykholeslami K, Schmerber S, Habiby Kermany M, Kaga K (2004) Vestibular-evoked myogenic potentials in three patients with large vestibular aqueduct. Hear Res 190:161-168

17. Sohmer H, Freeman S, Geal-Dor M, Adelman C, Savion I (2000) Bone conduction experiments in humans - a fluid pathway from bone to ear. Hear Res 146:81-88
18. Sohmer H, Freeman S, Perez R (2004) Semicircular canal fenestration-improvement of bone- but not air-conducted auditory thresholds. Hear Res 187:105-110

19. Streubel SO, Cremer PD, Carey JP, Weg N, Minor LB (2001) Vestibular-evoked myogenic potentials in the diagnosis of superior canal dehiscence syndrome. Acta Otolaryngol [Suppl] 545:41-49

20. Watson SR, Halmagyi GM, Colebatch JG (2000) Vestibular hypersensitivity to sound (Tullio phenomenon): structural and functional assessment. Neurology 54:722-728 